\title{
PLATINA SOBRE NANOBASTÕES DE TIO 2 PARA A REAÇÃO DE HIDRÓLISE DA CELULOSE
}

\author{
M. M. C. de ARAÚJO ${ }^{1}$, N. J. A. de ALBUQUERQUE ${ }^{1}$, M. P. FIGUEREDO ${ }^{1}$, R. M. de ALMEIDA ${ }^{2}$ \\ e S. M. P. MENEGHETTI ${ }^{2}$ \\ ${ }^{1}$ Universidade Federal de Alagoas, Centro de Tecnologia \\ ${ }^{2}$ Universidade Federal de Alagoas, Instituto de Química e Biotecnologia \\ E-mail para contato: araujommc@gmail.com
}

\begin{abstract}
RESUMO - A utilização do $\mathrm{TiO}_{2}$ em escala nanométrica proporciona modificações em suas propriedades que podem influenciar na atividade catalítica. Este trabalho investigou a atividade e seletividade catalítica do $\mathrm{TiO}_{2}$ e $\mathrm{Pt} / \mathrm{TiO}_{2}$ frente a conversão da celulose. Assim, foram sintetizados dois sistemas catalíticos: nanobastões de $\mathrm{TiO}_{2}\left(\mathrm{TiO}_{2}\right.$-nb) e nanobastões de $\mathrm{TiO}_{2}$ impregnados com Platina $\left(\mathrm{Pt} / \mathrm{TiO}_{2}-\mathrm{nb}\right)$. Os catalisadores foram caracterizados por IR, Raman, BET, DRX e MET. A partir das imagens de MEV foi confirmada a obtenção de nanobastões. A conversão da celulose sobre $\mathrm{TiO}_{2}$-nb foi de aproximadamente $10 \%$. A adição de platina proporcionou maior atividade catalítica, 20\%. Em termos de seletividade, o uso do $\mathrm{TiO}_{2}$-nb favoreceu a formação frutose, enquanto que o catalisador $\mathrm{Pt} / \mathrm{TiO}_{2}$-nb direciona a formação de glicose. O potencial do sistema $\mathrm{Pt} / \mathrm{TiO}_{2}$-nb pode ser atribuído ao efeito sinérgico da interação metal-suporte.
\end{abstract}

\section{INTRODUÇÃO}

Devido suas propriedades superficiais e eletrônicas únicas, o $\mathrm{TiO}_{2}$ possui uma grande versatilidade industrial, com aplicações em cosméticos, células solares, eletroquímica, catálise e outros. Materiais em escala nanométrica tem atraído bastante atenção nas últimas décadas, devido estes apresentarem propriedades físicas e químicas de interesse tecnológico quando suas dimensões são reduzidas. Diversos tipos de nanomateriais de $\mathrm{TiO}_{2}$ são reportados na literatura, incluindo nanopartículas, nanotubos, nanofios e os nanobastões. O método sintético mais empregado para a síntese dos nanobastões de $\mathrm{TiO}_{2}$ é a síntese hidrotermal, pois ela promove diferentes morfologias com excelente seletividade e eficiência, a depender das condições de síntese empregadas. Entretanto, o método é complicado e restrito a uma elevada pressão de reação, fazendo necessário o uso de uma autoclave. Bao et al (2007) desenvolveram um método simples e de baixo custo para a síntese de nanobastões de $\mathrm{TiO}_{2}$ em escala industrial. $\mathrm{O}$ método envolve a síntese dos bastões a partir do precurssor comercial sobre uma mistura eutética de hidróxidos fundidos, seguido da neutralização e calcinação.

O uso de óxidos metálicos contendo Platina ancorada, em reações de hidrogenólise da celulose têm sido bastante difundido na literatura. Fukuoka e Dephe (2006) mostraram que a presença da Platina direciona uma maior seletividade a açucares fermentáveis, podendo está relacionada à sua natureza ácida. 
Com base nestes conhecimentos, este trabalho, investiga a avaliação catalítica de $\mathrm{TiO}_{2}$-nb e $\mathrm{Pt} / \mathrm{TiO}_{2}$-nb, frente a reação de hidrólise da celulose em produtos de interesse industrial.

\section{EXPERIMENTAL}

\subsection{SÍNTESE DOS NANOBASTÕES DE $\mathrm{TiO}_{2}\left(\mathrm{TiO}_{2}\right.$-nb)}

Em um Becker de teflon foi adicionado uma mistura equimássica de hidróxido de sódio e hidróxido de potássio. Em seguida foi adicionado 1 g de $\mathrm{TiO}_{2}$ (comercial, Vetec) e submetido a 200 ${ }^{\circ} \mathrm{C}$ por 3 horas. Passado esse tempo, o Becker foi agitado para promover a homogeneização do meio, tendo em vista que a mistura de hidróxidos agora se encontrava no estado líquido, atuando como solvente. Após a agitação a mistura reacional foi mantida a $200{ }^{\circ} \mathrm{C} / 24 \mathrm{~h}$. Em seguida, o material resultante foi lavado com uma solução de $\mathrm{HCl} 0,1 \mathrm{M}$ sob filtração a vácuo. Por fim, o material foi sinterizado ao ar a $500{ }^{\circ} \mathrm{C} / 4 \mathrm{~h}$ com taxa de $5{ }^{\circ} \mathrm{C} / \mathrm{min}$, resultando num pó branco. Esse método sintético foi baseado nos trabalhos de Bao et al (2007).

\subsection{IMPREGNAÇÃO DE NANOPARTÍCULAS DE PLATINA NOS NANOBASTÕES DE $\mathrm{TiO}_{2}\left(\mathrm{Pt} / \mathrm{TiO}_{2}\right.$-nb)}

Dispersou-se $500 \mathrm{mg}$ de $\mathrm{TiO}_{2}$-nb (sintetizados previamente) em $97 \mathrm{ml}$ de uma solução de $\mathrm{H}_{2} \mathrm{PtCl}_{6} \cdot 6 \mathrm{H}_{2} \mathrm{O}$ a $2,6 \mathrm{mM}$ (correspondendo $10 \%$ em massa ao $\mathrm{TiO}_{2}$ ). Em seguida, a mistura foi colocada no ultrassom por 15 minutos, para promover uma maior dispersão dos bastões. Logo após, a suspensão foi colocada sob agitação magnética por 3 horas. Para promover a redução da platina, após esse período de tempo, foram adicionados $24,2 \mathrm{ml}$ de uma solução de $\mathrm{NaBH}_{4}$ a $0,04 \mathrm{M}$, onde ocorreu a mudança de coloração do meio, passando de amarelo para preto. A suspensão foi deixada sob agitação por mais 4 horas. Por fim, o material foi filtrado e seco na estufa a $90{ }^{\circ} \mathrm{C}$ por 24 horas. A impregnação da platina foi baseada nos trabalhos de Hua et al (2013).

\subsection{CARACTERIZAÇÃO DOS CATALISADORES}

A caracterização estrutural e textural dos nanobastões de $\mathrm{TiO}_{2}$ obtidos pelos métodos citados foi realizada utilizando os seguintes métodos:

- Difração de raio-X (DR-X) utilizando o difratômetro da ShimadzuXRD-6000.

- Análise superficial por fisissorção de $\mathrm{N}_{2}$ utilizando um Quantachrome Nova 4200e.

- Microscopia eletrônica de transmissão (MET) utilizando um microscópio FEI de 200kV, modelo Tecnai20, do Centro de Tecnologias Estratégicas do Nordeste (CETENE). 


\subsection{AVALIAÇÃO CATALÍTICA}

As reações de hidrólise da celulose foram realizadas em batelada, num reator de aço inoxidável de $200 \mathrm{~mL}$, acoplado a um manômetro. O reator foi colocado diretamente sobre um sistema de agitação magnética e o aquecimento foi realizado por um sistema com controlador de temperatura da marca Novus, o qual controla o tempo e a taxa de aquecimento. A quantificação dos produtos reacionais foi realizada por cromatografia líquida de alta eficiência (CLAE).

Foram realizados testes preliminares dos catalisadores a base de $\mathrm{TiO}_{2}$ sintetizados de forma a avaliar sua atividade e seletividade frente a reação de hidrólise da celulose. As condições reacionais utilizadas são listadas na Tabela 3. A escolha da temperatura e os teores de catalisador usados neste trabalho foi baseada no artigo de Dhepe e Fukuoka (2007), para fins comparativos.

Tabela 1 - Condições reacionais utilizadas na hidrólise da celulose.

\begin{tabular}{c|c|c|c|c}
\hline Celulose $(\mathbf{g})$ & Catalisador $(\mathbf{m o l})$ & Água $(\mathbf{m L})$ & Temperatura $\left({ }^{\circ} \mathbf{C}\right)$ & Tempo (h) \\
\hline 0,480 & $2,69 \times 10^{-5}$ & 70 & 190 & 4 \\
\hline
\end{tabular}

\section{RESULTADOS E DISCUSSÕES}

\subsection{DRX}

De forma a avaliar as características estruturais do $\mathrm{TiO}_{2}$ depois da síntese dos bastões e após a da impregnação da Platina, foram feitas análises de Difração de raio-X (Figura 1). A identificação da fase cristalina foi feita em comparação com os perfis de difração do banco de dados de padrões de difração para estruturas cristalinas JCPDS.

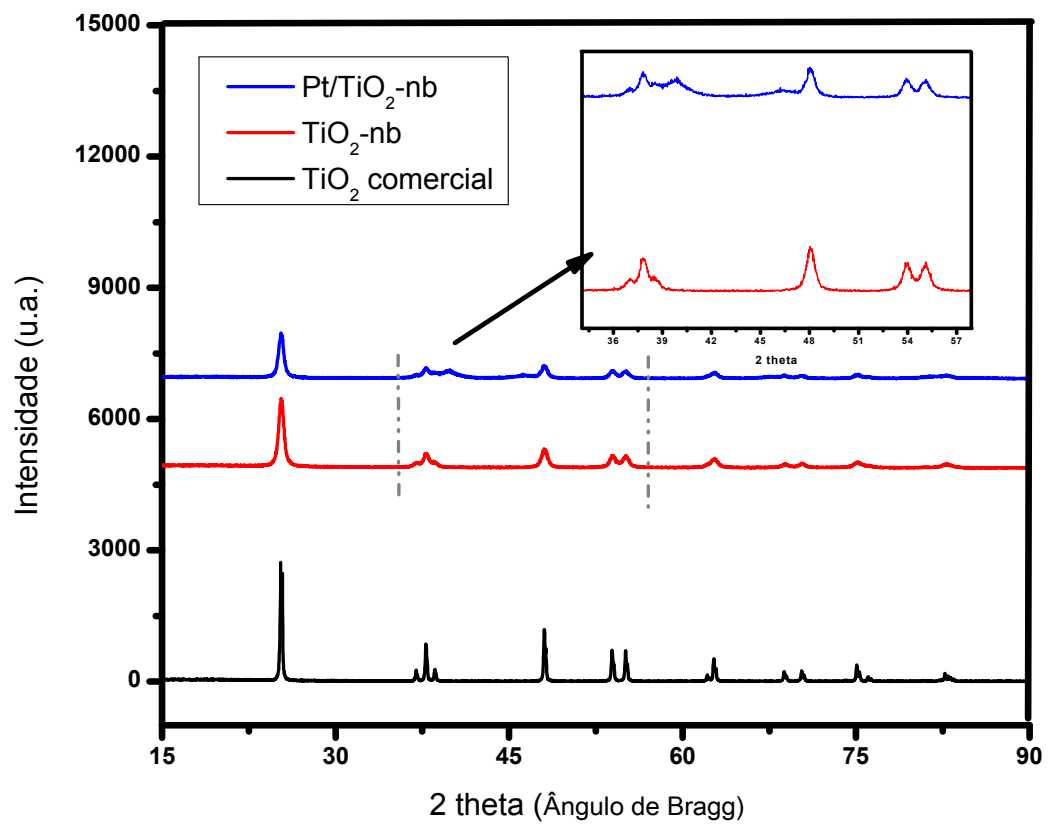

Figura 1 - Difratogramas de Raio-X para o $\mathrm{TiO}_{2}$ comercial, em forma de bastões $\left(\mathrm{TiO}_{2}\right.$-nb) e após a impregnação de Platina $\left(\mathrm{Pt} / \mathrm{TiO}_{2}-\mathrm{nb}\right)$. 
Através do difratograma, se pode afirmar que o $\mathrm{TiO}_{2}$ comercial se encontra na fase anatase, evidenciado pela presença de picos de grande intensidade em $25^{\circ}$ e em $48^{\circ}$. Após a síntese dos bastões $\left(\mathrm{TiO}_{2}-\mathrm{nb}\right)$, verificou-se a não alteração dos picos. Com a impregnação das nanopartículas de Platina, observa-se a presença de picos em $40^{\circ}$ e $46^{\circ}$, confirmando a presença das mesmas.

\subsection{FISISSORÇÃO DE $\mathbf{N}_{2}$}

A área superficial específica e distribuição do tamanho dos poros de um catalisador são alguns dos fatores mais importantes influenciam a atividade catalítica. Por conseguinte, uma área supercial elevada e um controle do tamanho e volume de poros irá conduzir a uma melhoria do desempenho catalítico.

Através dos dados obtidos por fisissorção de $\mathrm{N}_{2}$, observa-se que os nanobastões de $\mathrm{TiO}_{2}$, antes e após a impregnação, apresentam isotermas de adsorção/dessorção com histerese típica de sólidos mesoporosos do tipo III (Figura 2), de acordo com a classificação geral da IUPAC.

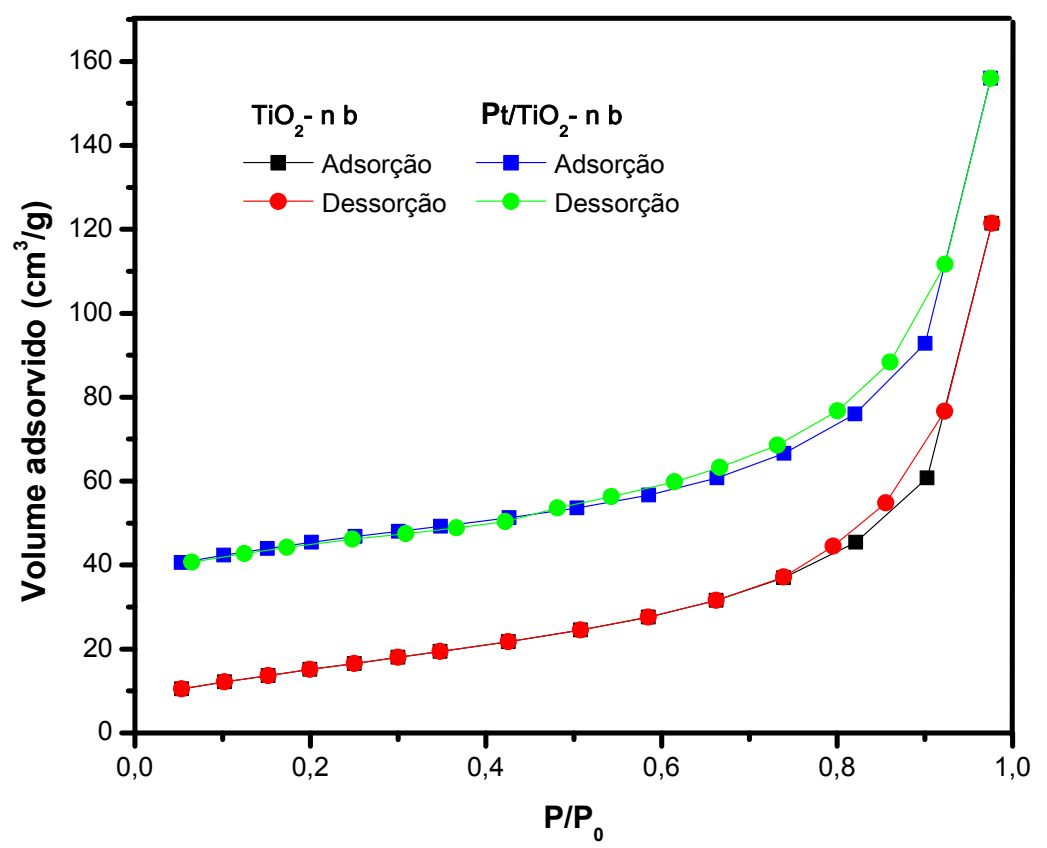

Figura 2 - Isotermas de adsorção/dessorção para os catalisadores $\mathrm{Pt} / \mathrm{TiO}_{2}$-nb e $\mathrm{TiO}_{2}$-nb

Os dados da isoterma do catalisador foram analisados para calcular a área superficial específica de BET $\left(S_{\mathrm{BET}}\right)$, o volume total de poros $\left(\mathrm{V}_{\mathrm{p}}\right)$, em função do volume total de nitrogênio adsorvido num ponto próximo à pressão de saturação, e o tamanho médio de poros $\left(\mathrm{D}_{\mathrm{p}}\right)$, onde $\mathrm{D}_{\mathrm{p}}=$ $4 \mathrm{~V}_{\mathrm{p}} / \mathrm{S}_{\mathrm{BET}}$ (Tabela 1$)$. 
Tabela 2 - Parâmetros estruturais dos catalisadores a base de $\mathrm{TiO}_{2}$ analisados por fisissorção de $\mathrm{N}_{2}$

\begin{tabular}{cccc}
\hline Catalisador & $\mathbf{S}_{\mathbf{B E T}}\left(\mathbf{m}^{2} / \mathbf{g}\right)$ & $\mathbf{V}_{\mathbf{p}}\left(\mathbf{c m}^{\mathbf{3}} / \mathbf{g}\right)$ & $\mathbf{D}_{\mathbf{p}}(\mathbf{\AA})$ \\
\hline $\mathrm{TiO}_{2}$ Comercial & 12 & 0,033 & 32 \\
$\mathrm{TiO}_{2}-\mathrm{nb}$ & 51 & 0,178 & 36 \\
$\mathrm{Pt} / \mathrm{TiO}_{2}-\mathrm{nb}$ & 57 & 0,189 & 36 \\
\hline
\end{tabular}

Analisando os dados da Tabela 1, observa-se que a transformação do $\mathrm{TiO}_{2}$ em nanobastões proporcionou um aumento na área superficial em cerca de 4 vezes. Outro dado interessante é o volume de poros, que chegou a quintuplicar após a formação dos bastões.

A distribuição do tamanho de poros dos catalisadores foi determinada pelo método de Barret, Joyner e Halenda (BJH), do qual se utiliza a equação de Kelvin, onde se verifica que o tamanho da grande maioria dos poros, para os catalisadores a base de nanobastões de $\mathrm{TiO}_{2}$ está na faixa de $30 \mathrm{a}$ $125 \AA$.

\subsection{MICROSCOPIA ELETRÔNICA DE TRANSMISSÃO (MET)}

A Figura 3 apresenta as imagens obtidas por MET dos nanobastões de $\mathrm{TiO}_{2}$, antes e após a impregnação da Platina. É possível verificar a formação dos bastões e que as nanopartículas de Pt encontram-se dispersas sobre os nanobastões. Utilizando as imagens de MET foram realizadas as medições das dimensões dos bastões e das partículas de Platina (Tabela 2).

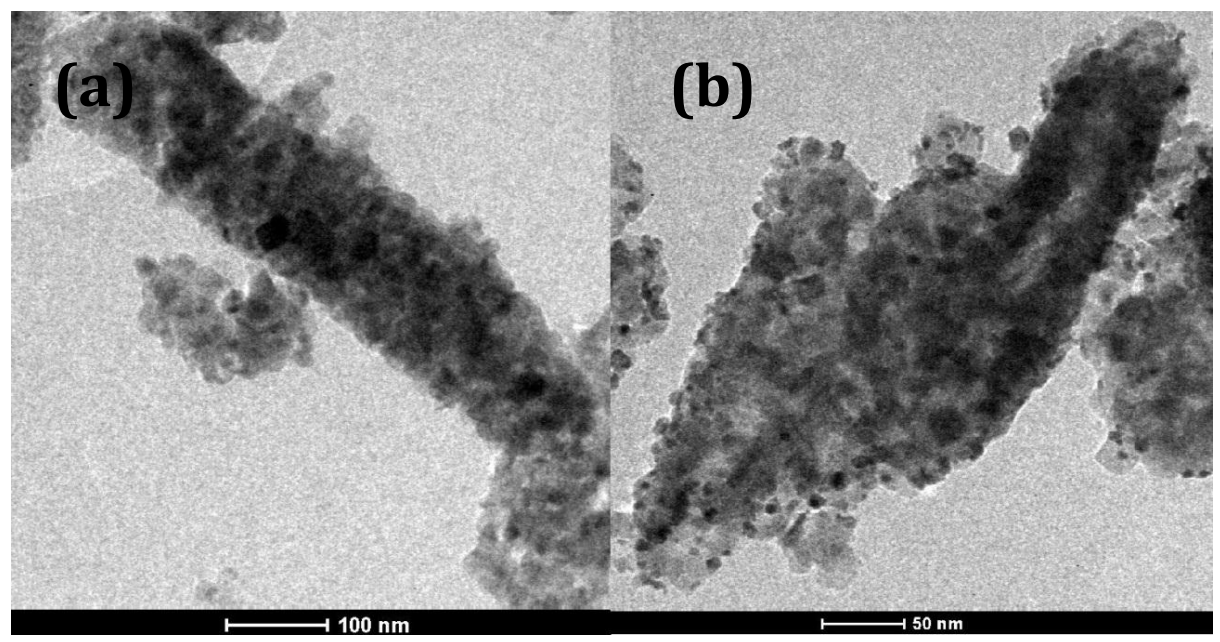

Figura 3 - Micrografia dos nanobastões de $\mathrm{TiO}_{2}$ : (a) $\mathrm{TiO}_{2}$-nb e (b) $\mathrm{Pt} / \mathrm{TiO}_{2}$-nb 
Tabela 3 - Análise do diâmetro das partículas por MET dos catalisadores sintetizados.
Catalisador
$\mathrm{N}^{\circ}$ de partículas
Nanobastões de $\mathrm{TiO}_{2}$
Nanopartículas de Pt medidas

Diâmetro médio (nm)

\begin{tabular}{cccc} 
& & Longitudinal | Transversal & Longitudinal \\
\hline $\mathrm{TiO}_{2}-\mathrm{nb}$ & 10 & $200 \mid 50$ & - \\
$\mathrm{Pt} / \mathrm{TiO}_{2}-\mathrm{nb}$ & 30 & $310 \mid 70$ & 6,3 \\
\hline
\end{tabular}

\subsection{AVALIAÇÃO CATALÍTICA}

Os resultados de conversão e seletividade para os testes catalíticos realizados utilizando as condições reacionais da Tabela 3 para diferentes catalisadores a base de $\mathrm{TiO}_{2}$ são mostrados na Tabela 4.

Através dos resultados de conversão da celulose (Tabela 4), vemos que a utilização do $\mathrm{Pt} / \mathrm{TiO}_{2}$-nb proporcionou uma conversão duas vezes maior que a dos outros catalisadores testados. Embora a conversão reacional utilizando $\mathrm{TiO}_{2}-\mathrm{nb}$ e $\mathrm{TiO}_{2}$ comercial seja, aproximadamente, a mesma, suas seletividades diferem. Enquanto o comercial direciona a formação preferencial de Glicose (14,5\%), o $\mathrm{TiO}_{2}$-nb promove a de frutose $(19,7 \%)$, o que pode está relacionado com a área superficial e o diâmetro de poros dos bastões, que são mais elevados.

Tabela 4 - Seletividade das reações de hidrólise celulose utilizando os catalisadores a base de $\mathrm{TiO}_{2}$ nas condições reacionais da Tabela 3 .

\begin{tabular}{|c|c|c|c|c|}
\hline \multicolumn{2}{|c|}{ Conversão (\%) } & $\begin{array}{c}\mathrm{TiO}_{2} \text { Comercial } \\
11\end{array}$ & $\begin{array}{c}\mathrm{TiO}_{2}-\mathrm{nb} \\
10\end{array}$ & $\begin{array}{c}\mathrm{Pt} / \mathrm{TiO}_{2}-\mathrm{nb} \\
20\end{array}$ \\
\hline \multirow{9}{*}{ Seletividade } & Celobiose & 4,8 & 2,3 & 3,3 \\
\hline & Glicose & 14,5 & 0,5 & 11,3 \\
\hline & Galactose & nd & 1,8 & nd \\
\hline & Frutose & 4,1 & 19,7 & 3,0 \\
\hline & Sorbitol & nd & 2,9 & nd \\
\hline & Ácido Lático & 1,9 & 0,6 & 0,9 \\
\hline & Anidro Glicose & 0,4 & nd & 0,7 \\
\hline & Ácido Fórmico & nd & nd & nd \\
\hline & HMF & nd & nd & 4,5 \\
\hline
\end{tabular}

nd = não detectado 


\section{9 a 22 de outubro de 2014 \\ Florianópolis/SC}

Os dados apresentados mostram o potencial catalítico dos sistemas sintetizados. É visto que o catalisador $\mathrm{TiO}_{2}$-nb apresentou uma conversão de $10 \%$, sendo $20 \%$ deste total frutose. Já o $\mathrm{Pt} / \mathrm{TiO}_{2}$-nb apresentou uma maior eficiência na despolimerização da celulose, convertendo cerca de $20 \%$, sendo $11 \%$ glicose.

Comparando com os resultados apresentados por Dhepe e Fukuoka (2007), vemos que os catalisadores a base de nanobastões de $\mathrm{TiO}_{2}$, apresentaram atividades similares aos catalisadores testados pelos autores, sendo que utilizando um tempo reacional bem menor (4h) e sem pressurização com $\mathrm{H}_{2}$.

Deste modo, pode-se afirmar que a presença da Platina aos nanobastões, promoveu uma sinergia propiciando um maior consumo da celulose e maior seletividade a glicose, dificultando a isomerização da mesma nestas condições reacionais.

\section{CONCLUSÃO}

Os materiais sintetizados demostraram atividade e seletividade frente a reação de conversão da celulose, com destaque para o $\mathrm{Pt} / \mathrm{TiO}_{2}$-nb. Visto que os poucos trabalhos reportados apresentam resultados inferiores em conversão aos apresentados nesse trabalho, estes catalisadores se mostram bastante promissores.

Tendo em vista que apenas testes catalíticos preliminares foram realizados, estudos estão em andamento para encontrar a condição ótima de reação para este sistema. Além disso, algumas análises de caracterização dos materiais estão em andamento para melhor nos certificamos de sua natureza e, assim, correlacionar os resultados.

\section{AGRADECIMENTOS}

UFAL, GCAR, PRH-40, ANP, PPGEQ, CNPQ E CAPES.

\section{REFERÊNCIAS}

BAO S.; BAO Q.; LI C.; DONG Z.Novel porous anatase $\mathrm{TiO}_{2}$ nanorods and their high lithium electroactivity, Electrochemistry Communications, 9, 1233-1238, 2007.

CHENX.; MAO S. Titanium Dioxide Nanomaterials: Synthesis, Properties, Modifications, and Applications. Chem. Rev. 107, 2891-2959, 2007.

DEPHE P.; FUKUOKA A.; Cracking of Cellulose Over Metal Supported Catalyst. Catal Surv Asia, v. 11, p. 186-191, 2007.

DEPHE P. FUKUOKA A.; Catalytic Conversion of Cellulose into Sugar Alcohols. Angew. Chem. Int. Ed., 45, 5161 -5163, 2006.

DIAK M.; GRABOWSKA E.; MARCHELEK M. ZALESKA A.; Decahedral TiO2 withexposedfacets: Synthesis, properties, photoactivity and applications. Applied Catalysis B: Environmental, v. 156-157, p. 213-235, 2014. 
HUA H.; HU C.; ZHAO Z.; LIU H.; XIE X.;XI Y. Pt nanoparticles supported on submicrometer sized $\mathrm{TiO}_{2}$ spheres for effective methanol and ethanol oxidation. Electrochimica Acta, 105, 130136, 2013.

LIUA N.; CHENA X.; ZHANGB J.; SCHWANKA J. A review on $\mathrm{TiO}_{2}$-based nanotubos synthesized via hydrothermal method: Formation mechanism, structure modification, and photocatalytic applications. Catalysis Today, v. 225, p. 34-51, 2014. 